\title{
Alicaforsen: An Emerging Therapeutic Agent for Ulcerative Colitis and Refractory Pouchitis
}

\author{
Terron Anthony Hosten ${ }^{\mathrm{a}, \mathrm{b}}$, Ke Zhao ${ }^{\mathrm{a}}$, Hong Qiu Han ${ }^{\mathrm{a}}$, \\ Gang Liu ${ }^{\mathrm{a}}$, Xiang Hui He ${ }^{\mathrm{a}}$
}

\begin{abstract}
Pouchitis is a relatively common complication that develops following ileal pouch-anal anastomosis in patients with complicated ulcerative colitis (UC). Both pouchitis and UC share similarities in their development, as well as in the mechanisms involving mediators of the inflammatory process. In the recent years, the discovery and investigation of biological therapies have led to advancement in the management of these disorders, and the continuation of research on this novel area holds strong implications for a future reduction in the use of invasive surgical procedures. Alicaforsen represents one of these emerging therapeutic agents, and has demonstrated promising results in both preclinical and clinical settings. This article reviews the therapeutic effects of alicaforsen for the management of UC and refractory pouchitis, with special emphasis on the mechanism of action of this therapeutic agent and the clinical studies asserting its effectiveness.
\end{abstract}

Keywords: Alicaforsen; Refractory pouchitis; Ulcerative colitis

\section{Introduction}

Ulcerative colitis (UC) continues to be a major illness affecting the quality of life of many patients worldwide. Not only this condition slowly growing in prevalence, but also the complications of surgical treatment, especially pouchitis, have become a growing issue of concern. Ileal pouch-anal anastomosis (IPAA) following total proctocolectomy (TPC) is considered as the standard surgical procedure for patients with UC, refractory to medical therapy $[1,2]$.

The formation of an ileal reservoir or pouch during

Manuscript accepted for publication March 3, 2014

${ }^{a}$ Department of General Surgery, Tianjin Medical University General Hospital, Tianjin, China

${ }^{b}$ Corresponding author: Terron Anthony Hosten, International Students'

Building, Tianjin Medical University, 22 Qi Xiangtai Rd, Heping

District, Tianjin 300070, China. Email: tahosten659@gmail.com

doi: http://dx.doi.org/10.14740/gr599w
IPAA, often results in inflammatory changes similar to that of UC [3, 4]. These changes give rise to pouchitis, which is now considered as the most common long term inflammatory complication [1, 3, 5-7]. According to statistics, approximately $30-45 \%$ of patients with UC must undergo surgical treatment at some point for this condition [8]. Studies carried out in China estimate a prevalence of UC of up to 11.6 cases per 100,000, as well as over a three-fold increase in the number of cases between the 1980s and 1990s [9]. Likewise, other studies show that the incidence of a first episode of pouchitis increases with time, and that $50 \%$ of the patients with recurring episodes develop refractory pouchitis [7].

With increased knowledge and better understanding about the pathophysiological mechanisms involved in the inflammatory process of UC and pouchitis, a series of biological agents have been developed over the past decade [5, $10,11]$. These agents include an antisense oligonucleotide called alicaforsen, which has been designed to inhibit the expression of adhesion molecules involved in the inflammatory cascade of UC and pouchitis [11, 12].

\section{UC and Pouchitis}

UC is categorized as an inflammatory bowel disease (IBD). It is a complex, chronic disease that is characterized by recurring, relapsing and remitting acute ulcerating inflammation of the colorectal mucosa. UC is known for its diffuse, continuous nature and its tendency to be restricted to the colon and rectum. Patients with UC, who fail medical therapy or develop complications such as dysplasia, toxic megacolon, bowel perforation and cancer, eventually require total TPC with IPAA. This surgical procedure involves the removal of the colon and rectum and the construction of a small bowel reservoir (pouch) that is subsequently anastomosed to the anus to reestablish the gastrointestinal tract [1, 8, 13-15].

Although TPC-IPAA is associated with improved quality of life of patients, inflammation of the constructed pouch (pouchitis) is not uncommon. In fact, pouchitis is considered as the most common long term inflammatory complication associated with this surgical procedure [1, 3, 5-8]. According to Shen et al, pouchitis is defined as a nonspecific inflamma- 


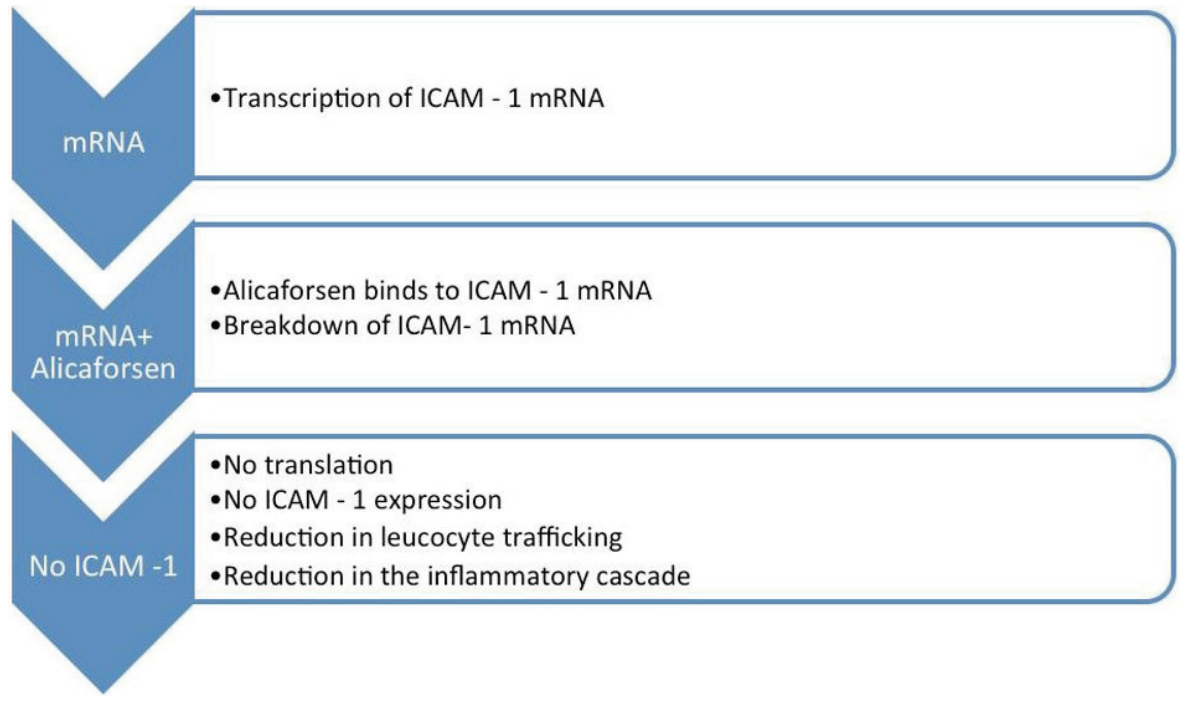

Figure 1. Cascade of events in the inflammatory process.

tory condition in the ileal pouch reservoir [1]. Its development is believed to be associated with contributing factors such as bacterial invasion and faecal stasis [5]. Pouchitis is diagnosed using a combination of clinical symptoms, endoscopic features of acute inflammation and histological evidence of a prominent polymorphonuclear cell exudate [3]. Conventional medical treatment of pouchitis includes antibiotics, steroids and aminosalicylates. This therapy, however, has proven less satisfactory for patients with chronic, recurrent and unremitting pouchitis. Patients with refractory pouchitis eventually undergo further surgery to remove the diseased pouch and resort to the use of a permanent ileostomy [7].

\section{Intercellular Adhesion Molecule-1 (ICAM-1)}

Human ICAM-1 forms part of the immunoglobulin superfamily $[12,16]$. It is essentially an inducible transmembrane glycoprotein that is expressed on the membranes of the colonic and vascular endothelium, as well as on the cell surface of a subset of leucocytes [12, 16-18]. ICAM-1 expression is

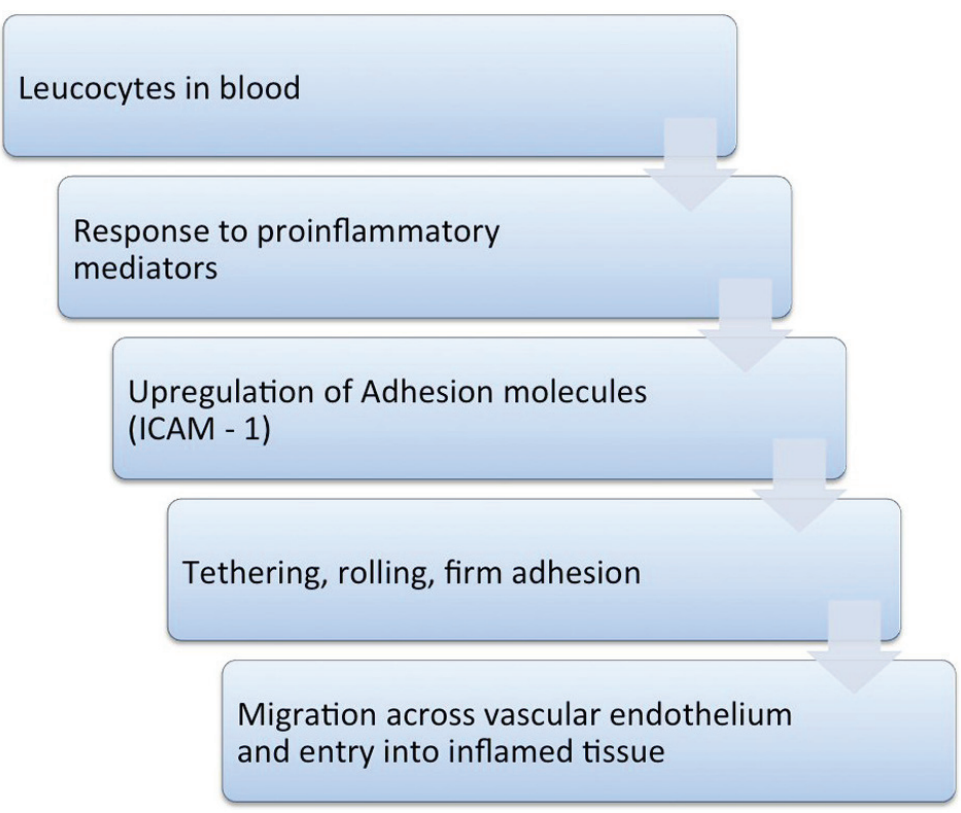

Figure 2. Mechanism of action of alicaforsen. 
Table 1. Studies Analyzing the Use of Alicaforsen in Pouchitis and Ulcerative Colitis

\begin{tabular}{|c|c|c|c|c|}
\hline \multirow{2}{*}{ References } & \multirow{2}{*}{ Year } & \multirow{2}{*}{$\begin{array}{l}\text { Number of } \\
\text { patients }\end{array}$} & \multicolumn{2}{|r|}{ Results } \\
\hline & & & Pouchitis & Ulcerative colitis \\
\hline Miner et al [7] & 2004 & 12 & $58 \%$ remission & N/A \\
\hline Van Deventer et al [16] & 2004 & 40 & N/A & $70 \%$ improvement in disease activity index \\
\hline Miner et al [21] & 2006 & 105 & N/A & $\begin{array}{l}24 \% \text { complete mucosal healing in } 240 \mathrm{mg} \\
\text { Alicaforsen group }\end{array}$ \\
\hline Miner et al [20] & 2006 & 15 & N/A & $\begin{array}{c}46 \% \text { improvement in disease activity index, } 33 \% \text { rate } \\
\text { of remission }\end{array}$ \\
\hline Van Deventer et al [12] & 2006 & 112 & $\mathrm{~N} / \mathrm{A}$ & Low relapse rate \\
\hline
\end{tabular}

upregulated in response to proinflammatory mediators, and this glycoprotein functions by binding to primary counterligands on the surface of leucocytes, such as $\beta_{2}$ integrins, Mac-1 and leucocyte function-associated antigen $1[12,16]$. Studies have demonstrated increased ICAM-1 expression within the inflamed gut, as well as an increase in circulating concentrations in the blood [16].

\section{Pathophysiology of the Inflammatory Process in UC and Pouchitis}

According to Lightfoot et al, ICAM-1 is responsible for the migration of leucocytes from the areas of inflammation, and it constitutes an active component in the pathophysiology of inflammatory bowel disease [17].

Leucocyte trafficking in the intestines occurs as a result of the expression of ICAM-1. In order to reach the area of inflammation, leucocytes must be guided by a pathway of adhesion molecules. ICAM-1, along with other similar adhesion molecules, interacts with the counter-ligands on the surface of leucocytes, thereby contributing to the migration of these cells [10]. The first step involves their interaction with the endothelium of the post capillary vessels. In order to slow down the leucocytes that are normally travelling at relatively high speed, the adhesion system is activated, causing them to tether and roll along the endothelium. These processes are subsequently followed by firm adhesion and later migration of the leucocytes from the blood into the inflamed tissue [10, 13, 14, 16] (Fig. 1).

The expression of ICAM-1 is upregulated in response to proinflammatory mediators. This process also occurs along the epithelium of the colon, which further aggravates the lo- cal inflammatory process [16] (Fig.1).

\section{Alicaforsen}

The advances in biotechnology, as well as in the research of the pathophysiological mechanisms involved in the inflammatory process of inflammatory bowel disease, have led to the discovery and investigation of a group of biological agents that are capable of inhibiting the expression of molecules that give rise to the inflammatory cascade, especially in UC and pouchitis [3, 10-12].

Alicaforsen constitutes one of the most promising biological agents for the treatment of both UC and refractory pouchitis $[7,10,12,16,18]$. It is a 20-base antisense oligonucleotide (5'-GCCCAAGCTGGCATCCGTCA-3') developed by ISIS pharmaceuticals Inc. (Carlsbad, CA, USA), and assigned the code name ISIS 2302 [11-13, 18]. Alicaforsen is considered as a highly selective ICAM-1 inhibitor that down-regulates ICAM-1 mRNA, the messenger RNA responsible for the development and expression of ICAM-1 on the cell surface. ICAM-1 plays a very important role in the inflammatory process associated with UC and pouchitis $[7,11-13,16]$.

\section{Mechanism of Action of Alicaforsen}

Alicaforsen is known as an antisense oligonucleotide, because its base sequence is complimentary to the ICAM-1 messenger RNA [19]. Following the transcription of the ICAM-1 mRNA, alicaforsen binds with the same through a process called hybridization. This process renders the 
ICAM-1 mRNA ineffective and consequently results in its destruction by cleavage $[7,10,12,16]$.

As a result, translation of ICAM-1 mRNA and subsequent ICAM-1 expression are inhibited. This entire chain of events reduces the migration and trafficking of leucocytes, with a substantial reduction in the inflammatory cascade associated with UC and refractory pouchitis [7, 10, 14, 18] (Fig. 2).

\section{Studies Asserting the Effectiveness of Alica- forsen}

Over the past recent years, clinical studies have been undertaken to assert the effectiveness of the use of alicaforsen in patients with UC and refractory pouchitis. Overall, the results are promising, although more clinical trials in various centers are still required [7, 10, 12, 16, 20, 21] (Table 1).

Miner et al carried out an open label uncontrolled study of 12 patients with chronic, unremitting pouchitis. The subjects were treated with $240 \mathrm{mg}$ of alicaforsen enema for 6 weeks. Overall, results showed a $58 \%$ remission of pouchitis, with no serious side effects of the formulation [7] (Table 1).

Likewise, Miner et al carried out a randomized, doubleblind, active controlled trial, with the objective of evaluating the safety and efficacy of two dose formulations of alicaforsen enema, compared with mesalazine enema for the treatment of mild to moderate left-sided UC. A total of 55 subjects received a nightly enema of $120 \mathrm{mg}$ alicaforsen, while another 50 subjects received $240 \mathrm{mg}$ of the same drug. The treatment lasted for 6 weeks, followed by a 24-week monitoring period. Another group of 54 subjects received a $4 \mathrm{~g}$ mesalazine therapy, with the same treatment regime and duration. Results showed that the median duration of response to alicaforsen enema treatment was two to threefold longer in comparison with that of mesalazine. A $24 \%$ complete mucosal healing rate was recorded in the $240 \mathrm{mg}$ alicaforsen group, in comparison with a $17 \%$ healing rate in the mesalazine group [21] (Table 1).

Miner et al went on to investigate the bioavailability and therapeutic activity of alicaforsen, administered as a rectal enema to subjects with UC, using an open-label study. In this study, 15 subjects were administered $240 \mathrm{mg}$ nightly doses of alicaforsen for 6 weeks. Results obtained showed that the concentrations of the intact oligonucleotide in mucosal colonic tissue biopsy were significantly higher than those observed in plasma. A $46 \%$ reduction in main disease activity index, as well as a $33 \%$ rate of remission was observed at the end of the period of treatment [20] (Table 1).

Van Deventer et al carried out a randomized, controlled, double-blind, escalating dose study of alicaforsen enema in 40 patients with active UC. Results showed a 70\% improvement in the disease activity index of the patients treated with alicaforsen as compared to a $28 \%$ response in patients with placebo. In addition, the results improved in a dose dependant manner, with a high level of statistical significance [16] (Table 1).

Similarly, a phase II dose ranging, double-blind, placebo controlled study of alicaforsen enema was carried out by Van Deventer et al in subjects with left-sided UC. A low relapse rate was recorded, with adequate tolerance to study dosing [12] (Table 1).

\section{Application of Alicaforsen}

According to Dike et al, alicaforsen has received fast-track approval from the Food and Drug Administration (FDA), taking into consideration the significant results obtained in the clinical trials, as well as the safety profile demonstrated with its use [22, 23]. Alicaforsen has also received approval from the European Medicines Agency (EMA) for use throughout Europe. This drug is currently licensed to Atlantic Pharmaceuticals Limited [23].

Overall, the most widely employed method for the use of alicaforsen has been via retention enema. This method provides a direct interaction between alicaforsen and the inflamed mucosa $[7,12,16]$. According to Atlantic Healthcare, alicaforsen can be obtained for treatment, following specifications from an authorized healthcare professional. The recommended dosage is $240 \mathrm{mg}(60 \mathrm{~mL})$, once daily via enema. A total of 42 sessions constitute a full treatment course [24].

\section{Conclusions}

The management of UC and pouchitis continues to be a very important area of concern. The development of biological agents has paved the way for the use of new therapeutic strategies in the treatment of these disorders. Alicaforsen constitutes one of the most promising biological agents known to down-regulate the expression of ICAM-1, responsible for leucocyte migration and trafficking during the inflammatory response process. Multicenter studies assert the effectiveness of alicaforsen as a promising therapeutic agent for UC and refractory pouchitis. Although alicaforsen has received fasttrack approval, more clinical trials in different centers are required to further validate its use.

\section{Acknowledgement}

The authors of this article would like to acknowledge and extend their heartfelt gratitude to the Department of General Surgery of the Tianjin Medical University General Hospital and the library services of the Tianjin Medical University, for providing assistance with the research of articles related 
to this topic.

\section{Conflict of Interest}

The authors declare that there is no conflict of interests regarding the publication of this article. Likewise, there are no financial issues to be disclosed.

\section{References}

1. Shen B, Lashner BA. Diagnosis and treatment of pouchitis. Gastroenterol Hepatol (N Y). 2008;4(5):355-361.

2. Penna C, Dozois R, Tremaine W, Sandborn W, LaRusso N, Schleck C, Ilstrup D. Pouchitis after ileal pouch-anal anastomosis for ulcerative colitis occurs with increased frequency in patients with associated primary sclerosing cholangitis. Gut. 1996;38(2):234-239.

3. Schouten WR. Pouchitis. Mediators Inflamm. 1998;7(3):175-181.

4. Ruseler-van Embden JG, Schouten WR, van Lieshout LM. Pouchitis: result of microbial imbalance? Gut. 1994;35(5):658-664.

5. Luukkonen P, Jarvinen H, Tanskanen M, Kahri A. Pouchitis--recurrence of the inflammatory bowel disease? Gut. 1994;35(2):243-246.

6. Zippi M, Cassieri C, Avallone EV, Pica R. Infliximab for the treatment of pouchitis. World J Clin Cases. 2013;1(6):191-196.

7. Miner P, Wedel M, Bane B, Bradley J. An enema formulation of alicaforsen, an antisense inhibitor of intercellular adhesion molecule-1, in the treatment of chronic, unremitting pouchitis. Aliment Pharmacol Ther. 2004;19(3):281-286.

8. Ianco O, Tulchinsky H, Lusthaus M, Ofer A, Santo E, Vaisman N, Dotan I. Diet of patients after pouch surgery may affect pouch inflammation. World J Gastroenterol. 2013;19(38):6458-6464.

9. Ye L, Cao Q, Cheng J. Review of inflammatory bowel disease in China. ScientificWorldJournal. 2013;2013:296470.

10. Triantafillidis JK, Merikas E, Georgopoulos F. Current and emerging drugs for the treatment of inflammatory bowel disease. Drug Des Devel Ther. 2011;5:185-210.

11. Yacyshyn BR, Chey WY, Goff J, Salzberg B, Baerg R, Buchman AL, Tami J, et al. Double blind, placebo controlled trial of the remission inducing and steroid sparing properties of an ICAM-1 antisense oligodeoxynucleotide, alicaforsen (ISIS 2302), in active steroid dependent Crohn's disease. Gut. 2002;51(1):30-36.
12. van Deventer SJ, Wedel MK, Baker BF, Xia S, Chuang E, Miner PB, Jr. A phase II dose ranging, doubleblind, placebo-controlled study of alicaforsen enema in subjects with acute exacerbation of mild to moderate left-sided ulcerative colitis. Aliment Pharmacol Ther. 2006;23(10):1415-1425.

13. Ghosh S, Panaccione R. Anti-adhesion molecule therapy for inflammatory bowel disease. Therap Adv Gastroenterol. 2010;3(4):239-258.

14. Murphy CT, Nally K, Shanahan F, Melgar S. Shining a light on intestinal traffic. Clin Dev Immunol. 2012;2012:808157.

15. Cho W, Cho YB, Kim JY, Chang DK, Kim YH, Kim $\mathrm{HC}$, Yun SH, et al. Outcome of total proctocolectomy with ileal pouch-anal anastomosis for ulcerative colitis. J Korean Surg Soc. 2012;83(3):135-140.

16. van Deventer SJ, Tami JA, Wedel MK. A randomised, controlled, double blind, escalating dose study of alicaforsen enema in active ulcerative colitis. Gut. 2004;53(11):1646-1651.

17. Lightfoot HL, Hall J. Target mRNA inhibition by oligonucleotide drugs in man. Nucleic Acids Res. 2012;40(21):10585-10595.

18. Mansoor M, Melendez AJ. Advances in antisense oligonucleotide development for target identification, validation, and as novel therapeutics. Gene Regul Syst Bio. 2008;2:275-295.

19. Morcos PA. Achieving targeted and quantifiable alteration of mRNA splicing with Morpholino oligos. Biochem Biophys Res Commun. 2007;358(2):521-527.

20. Miner PB, Jr., Geary RS, Matson J, Chuang E, Xia S, Baker BF, Wedel MK. Bioavailability and therapeutic activity of alicaforsen (ISIS 2302) administered as a rectal retention enema to subjects with active ulcerative colitis. Aliment Pharmacol Ther. 2006;23(10):14271434.

21. Miner PB, Jr., Wedel MK, Xia S, Baker BF. Safety and efficacy of two dose formulations of alicaforsen enema compared with mesalazine enema for treatment of mild to moderate left-sided ulcerative colitis: a randomized, double-blind, active-controlled trial. Aliment Pharmacol Ther. 2006;23(10):1403-1413.

22. Dike UA, Honeywell MS, Welsh TM, et al. Ulcerative colitis: therapeutic management update. US Pharm. 2010;35(12):HS 21-HS 26.

23. ISIS Pharmaceuticals. Drugs in Development: Alicaforsen, 2014. Retrieved from http://www.isispharm. com/Pipeline/Therapeutic-Areas/NeurodegenerativeDisease.htm\#Alicaforsen.

24. Atlantic Healthcare, "Alicaforsen," 2014. Retrieved from http://atlantichc.com/alicaforsen.htm. 